\title{
Impact of COMT Val I58Met on executive functioning in the context of HIV and methamphetamine
}

This article was published in the following Dove Press journal:

Neurobehavioral HIV Medicine

8 January 2010

Number of times this article has been viewed

\author{
Chad A Bousman' \\ Mariana Cherner' \\ Stephen J Glatt ${ }^{2}$ \\ J Hampton Atkinson' \\ Igor Grant ${ }^{\prime}$ \\ Ming T Tsuang \\ Ian P Everall' \\ 'Department of Psychiatry, University \\ of California San Diego, La Jolla, \\ California, USA; ${ }^{2}$ Department of \\ Psychiatry and Behavioral Sciences, \\ and Medical Genetics Research \\ Center, SUNY Upstate Medical \\ University, Syracuse, NY, USA
}

\begin{abstract}
The catechol-O-methyltransferease (COMT) Val allele has been linked to executive dysfunction among healthy individuals. The nature of this relationship is unknown in the context of HIV infection and/or methamphetamine (METH) dependence, two conditions that can alter dopaminergic system functioning. We sought to determine if the putative relationship between COMT and executive dysfunction could be observed among individuals with and without HIV-infection and/or METH dependence, and to explore the specificity of this relationship by examining other cognitive domains. Utilizing an existing cohort of 229 men with and without HIV infection and/or METH dependence we found that Met/Met carriers within the HIV-only and control groups, displayed better executive functioning compared to Val/Val and Val/Met carriers. However, this effect was attenuated in the METH-only and comorbid (ie, HIV+/METH+) groups. Examination of other neurocognitive domains were not consistent with effects found for executive functioning. Results support the presumed neuroprotective effect of Met/Met genotype on executive functioning among HIV-only and control groups. Among METH-only and comorbid groups, the slower rate of dopamine clearance conferred by the Met/Met genotype may increase the risk of adverse effects of METH, resulting in comparable executive dysfunction to that of Val allele carriers.
\end{abstract}

Keywords: Val158Met, Endophenotype, Executive Function

\section{Introduction}

Executive functions are a cluster of high-order cognitive abilities largely dependent on the integrity of the prefrontal cortex (PFC), which include decision-making, planning, and self-monitoring, as well as behavior initiation, organization, and inhibition. ${ }^{1}$ Both HIV infection and methamphetamine (METH) dependence can have adverse effects on general cognitive and executive functioning. ${ }^{2-8}$ These cognitive deficits among METH users and/or HIV-infected individuals may ultimately result in poor decision-making and subsequently may increase the probability of HIV transmission or adversely affect disease management. Thus, understanding the underlying biological (eg, genetic) mechanisms of cognitive abilities, specifically executive functioning, in different environmental contexts may provide avenues for future early identification and preventative treatments of cognitive impairment related to HIV and METH, and potentially inform interventions aimed at reducing the current rise in HIV-infection rates.

Dopaminergic neurotransmission in the PFC is known to influence cognitive capacity, and dopamine (DA) levels in the synapse can influence cognitive functioning. ${ }^{9,10}$ As aforementioned, both METH use and HIV-infection have negative effects on cognitive functioning but they have also been shown to alter cortical dopamine levels. METH has
Correspondence: Chad A Bousman Department of Psychiatry, University of California San Diego, I 40 Arbor Drive, San Diego, CA 92103, USA

Email cbousman@ucsd.edu 
long been established as a dopaminergic agonist that results in synaptic dopamine influxes acutely and intracellular dopamine deficits over long-term exposure; ${ }^{11,12}$ whereas, HIV-infection has been shown to lower dopamine levels in the cerebrospinal fluid ${ }^{13-15}$ and postmortem human brains. ${ }^{16}$ Thus, mechanisms that assist in removing dopamine from the synapse (such as catechol-O-methyltransferease (COMT)) play an important role in regulating dopamine levels in the brain and subsequently may moderate cognitive functioning. COMT is a major mammalian enzyme involved in the metabolic degradation of released DA. Although, noradrenaline transporters ${ }^{17}$ and monoamine oxidase $(\mathrm{MAO})^{18}$ contribute to elimination of DA, COMT accounts for more than $60 \%$ of the metabolism of DA in the PFC. ${ }^{19}$

Our primary interest in this study was a prevalent single nucleotide polymorphism (SNP) of COMT involving a Valine (Val) to Methionine (Met) amino acid substitution at codon 158 in the membrane-bound COMT (MB-COMT) or position 108 in the soluble-COMT (S-COMT). In human postmortem PFC, the $\mathrm{Val}$ allele at this locus is $40 \%$ more enzymatically active than the Met allele. ${ }^{20}$ Thus, carriers of the Val allele metabolize dopamine more efficiently, resulting in lower levels of DA in the synapse and ultimately a reduction in DA receptor activation. Recent research in transgenic and knockout mice ${ }^{21}$ have identified the integral role of COMT in cognition, and several human studies have echoed these results, ${ }^{22-26}$ albeit meta-analyses of human studies have reported mixed results. ${ }^{27,28}$ However, Mattay and colleagues ${ }^{29}$ proposed and tested a theoretical inverted U-shaped model of the relationship between DA activity in the PFC and cognitive performance in the context of laboratory-controlled acute amphetamine administration in healthy volunteers. Results of that study suggested that the relationship between COMT and PFC function is likely to be context-dependent and more complex than a simple dichotomy in which a Val allele is harmful and a Met allele is protective. To our knowledge, the putative context-dependent effect of COMT has not yet been examined in the natural (ie, nonlaboratory) context of abstinent methamphetamine dependence and HIV-infection.

The purpose of this study was to examine the presumed favorable effect of the COMT Met/Met genotype on executive functioning among recently abstinent METH-dependent and/or HIV-infected participants from an ethnically diverse population. We hypothesized that the contexts of both METH dependence and HIV-infection, two conditions that can alter dopaminergic systems, would modulate the relationship between COMT genotype and executive functioning. Secondarily, we explored the specificity of the hypothesized relationship by examining other cognitive functioning domains (ie, learning, recall, speed of information processing, motor speed, verbal fluency, and working memory).

\section{Methods}

\section{Participants}

Participants were volunteers evaluated at the HIV Neurobehavioral Research Center (HNRC) at the University of California in San Diego as part of a cohort study focused on central nervous system effects of HIV and methamphetamine. The current study comprised 229 men of Caucasian $(n=158)$, African-American $(n=31)$, and nonwhite Hispanic $(n=40)$ ethnicity. In an effort to examine the differential contextual effects of METH dependence and HIV-infection on the putative COMT and executive functioning relationship, participants were classified into one of the following four groups: methamphetamine-dependent/HIV-seropositive [METH+/HIV+; $n=58]$; methamphetamine-dependent/ HIV-seronegative [METH-only; $n=50]$; methamphetamine nonusers/HIV-seropositive [HIV-only; $n=72$ ); and methamphetamine nonusers/HIV-seronegative [controls; $n=49$ ]. All participants were seronegative for hepatitis $\mathrm{C}$ infection.

HIV serological status was determined by enzyme linked immunosorbent assays (ELISA) plus a confirmatory test. $\mathrm{METH}+$ participants met dependence criteria in their lifetime and abuse criteria within the previous 18 months, as determined by the Structured Clinical Interview for the Diagnostic and Statistical Manual of Mental Disorders Version IV (SCID). Potential participants were excluded if they: 1) had a medical (eg, diabetes) or neurologic (eg, epilepsy) confound attributable to a condition other than HIV disease or METH use that may result in confounding neuropsychological impairment or that involved dopaminergic disturbance, 2) had a diagnosis of schizophrenia, bipolar disorder, or another severe psychiatric illness that might make participation in the study problematic or unsafe, and/or 3) met lifetime dependence criteria for other drugs, unless the dependence was judged by a doctoral-level clinician to be remote (greater than 5 years ago) and episodic in nature. Alcohol dependence within the last year was also an exclusion criterion. Participants were not actively using other substances, with the exception of cannabis and alcohol. Use of any substances other than alcohol or cannabis within the last 10 days, alcohol use exceeding three ounces in the previous 24 hours, or any cannabis use in the previous 24 hours, resulted in deferral of assessment until these criteria were no longer met.

Participants with a history of METH dependence were primarily recruited from residential drug-treatment programs 
in the San Diego area, while those participants without a history of METH abuse were recruited from the larger San Diego community through the use of flyers and appearances at community events. All participants gave written consent prior to enrollment and all procedures were approved by the Human Research Protection Program of the University of California, San Diego and San Diego State University.

\section{Executive functioning, METH use, and HIV characterization}

Executive functioning was determined as part of a comprehensive battery of tests covering seven ability domains (Table 1). Raw scores for the component tests were converted to demographically-adjusted T-scores $(\mathrm{M}=50, \mathrm{SD}=10)$, including adjustments for age, education, gender, and ethnicity as available for each test. The demographicallyadjusted T-scores for each test were then converted into deficit scores, which reflect degree of impairment by setting performances within the normal range at zero with a range from 0 (T-score $>39$; no impairment) to 5 (T-score $<20$; severe impairment). Finally, the individual deficit scores were averaged within each domain to derive the domain deficit score, which reflects the severity of deficit within each particular domain. Previous work has demonstrated that deficit scores achieve good diagnostic agreement with classifications made by blind clinical ratings, with a cut point for impairment set at $\geq 0.50$. ${ }^{7,30}$ The executive functioning domain deficit score, of particular focus in this study, was made up of 1) perseverative responses on the Wisconsin Card Sorting Test; 2) errors on the Halstead Category Test, which measures abstraction and cognitive flexibility; and 3) time to complete the Trail Making Test part B, reflecting ability to switch and maintain attention between ongoing sequences. All neurocognitive testing and scoring was performed by trained psychometrists blinded to participants' genotypes.
Additional information for each participant was collected as it relates to a lifetime diagnosis of Major Depression Disorder (MDD), Bipolar Disorder I or II as well as abuse and/or dependence for sedatives, cannabis, opioids, cocaine, hallucinogens, and alcohol, using the SCID-IV. For METH+ participants, additional information was collected regarding age at first use, years and quantity of cumulative use, and days since last use of METH; whereas for HIV-infected participants, HIV RNA plasma copies were ascertained as part of a larger neuromedical evaluation.

\section{DNA extraction and genotyping}

DNA was extracted from peripheral blood mononuclear cells stored (three to five years) at $-70^{\circ} \mathrm{C}$ using the QIAamp DNA Mini kit (Qiagen, Valencia, CA). The COMT Val158Met polymorphism (rs4680) was assayed employing a Multiplex polymerase chain reaction (PCR) technique designed using Sequenom SpectroDESIGNER software (version 3.0.0.3) that has been described in more detail elsewhere ${ }^{31,32}$ All genotyping was performed by an accredited commercial laboratory (Harvard Medical School-Partners Healthcare Center for Genetics and Genomics, Cambridge, MA CLIA No. 22D1005307).

\section{Statistical analysis}

All statistical tests and procedures were conducted using SPSS 10.0 (SPSS Inc., Chicago, IL). Group comparisons on demographic data were performed using analysis of variance (ANOVA) or chi-square tests, depending on the distribution of the dependent variable. For this study, COMT genotype was dichotomized (Met/Met versus $\mathrm{Val} / \mathrm{Val}$ or $\mathrm{Val} / \mathrm{Met}$ ) for hypothesis-testing based on previous findings. ${ }^{23}$ Mean group differences in executive functioning and other neurocognitive domain performance were analyzed with Student's t-tests. Group differences in the proportions of impaired participants

Table I Neurocognitive test battery

\begin{tabular}{ll}
\hline Domain & Tests \\
\hline Learning & BVMT-R Learning, HVLT-R Learning, Story Memory Test-Learning, Figure Memory Test-Learning \\
Recall & BVMT-R Delayed Recall, HVLT-R Delayed Recall, Story Memory Test Retention, Figure Memory Test Retention \\
Attention/Working memory & PASAT Total Correct, WAIS-III Letter-Number Sequencing \\
Processing speed & Trail Making Test A,WAIS-III Symbol Search, WAIS-III Digit Symbol, Stroop Color-Word Incongruent \\
Executive functioning & Trail Making Test B, Halstead Category Test Errors, WCST-64 Perseveration \\
Motor speed & Grooved Pegboard Test \\
Verbal fluency & Letter Fluency (F.A.S.) \\
\hline
\end{tabular}

Abbreviations: BVMT-R, BriefVisual Memory Test-revised; HVLT-R, Hopkins Verbal Learning Test-revised; PASAT, Paced Auditory Serial Addition Test; WAIS, Wechsler Adult Intelligence Scale;WCST-64, Wisconsin Card Sort Test-64 Card Version. 
Table 2 Participant characteristics $(\mathrm{N}=229)$

\begin{tabular}{|c|c|c|c|c|c|}
\hline Characteristic & I & 2 & 3 & 4 & Post-hoc \\
\hline \multirow[t]{2}{*}{ All groups } & Control & HIV only & METH only & METH and HIV & \\
\hline & $(n=49)$ & $(n=72)$ & $(n=50)$ & $(n=58)$ & \\
\hline Age (years) M (sd) & $37(12)$ & $40(9)$ & $36(10)$ & $37(7)$ & \\
\hline Education (years) M (sd) & $13(2)$ & $13(2)$ & $13(2)$ & $13(3)$ & \\
\hline WRAT4 M (sd) & $103(1 \mathrm{I})$ & I0I (I0) & $96(12)$ & $99(\mathrm{II})$ & $\mathrm{I}, 2>3^{* *}$ \\
\hline \multicolumn{6}{|l|}{ Ethnicity (\%) } \\
\hline Caucasian & 21 & 29 & 26 & 25 & \\
\hline African-American & 32 & 48 & 0 & 19 & $\mathrm{I}, 2>3,4^{*}$ \\
\hline Hispanic & 15 & 30 & 23 & 33 & $2,3,4>I^{*}$ \\
\hline WCST perseverative errors (T-score) M (sd) & $46(12)$ & $47(15)$ & $4 \mid(\mid 3)$ & $46(15)$ & \\
\hline Category Test errors (T-score) M (sd) & $49(10)$ & $45(9)$ & $46(10)$ & $43(10)$ & $1>4^{*}$ \\
\hline Trails Making Test Part B (time) M (sd) & $52(\mathrm{II})$ & $50(10)$ & $47(10)$ & $47(\mathrm{II})$ & $1>4^{*}$ \\
\hline Executive functioning (deficit score) M (sd) & $0.38(0.61)$ & $0.55(0.67)$ & $0.64(0.74)$ & $0.65(0.79)$ & \\
\hline Executive functioning (\% impaired) & 17 & 43 & 50 & 52 & \\
\hline Major depression disorder (\% lifetime) & 20 & 44 & 33 & 49 & $2,3,4>I^{*}$ \\
\hline Bipolar I or II (\% lifetime) & 0 & 4 & 4 & 7 & \\
\hline \multicolumn{6}{|l|}{ Sedative (\% lifetime) } \\
\hline Abuse & 2 & 0 & 4 & 3 & \\
\hline Dependence & 0 & 0 & 0 & 0 & \\
\hline \multicolumn{6}{|l|}{ Cannabis (\% lifetime) } \\
\hline Abuse & 21 & II & 36 & 33 & $3,4>1,2^{* * 1}$ \\
\hline Dependence & 4 & 3 & 24 & 10 & $3,4>1,2^{* * *}$ \\
\hline \multicolumn{6}{|l|}{ Stimulant (\% lifetime) } \\
\hline Abuse & 4 & 2 & - & - & \\
\hline Dependence & 0 & 0 & 100 & 100 & $3,4>1,2^{* *}$ \\
\hline \multicolumn{6}{|l|}{ Opiod (\% lifetime) } \\
\hline Abuse & 0 & 0 & 6 & 5 & \\
\hline Dependence & 0 & 0 & 0 & 0 & \\
\hline \multicolumn{6}{|l|}{ Cocaine (\% lifetime) } \\
\hline Abuse & 2 & 4 & 19 & 15 & $3,4>1,2^{*}$ \\
\hline Dependence & 0 & 0 & 16 & 16 & $3,4>1,2^{* * *}$ \\
\hline \multicolumn{6}{|l|}{ Hallucinogen (\% lifetime) } \\
\hline Abuse & 4 & 3 & 16 & 7 & $3>1,2,4^{*}$ \\
\hline Dependence & 0 & 0 & 0 & 2 & \\
\hline \multicolumn{6}{|l|}{ Alcohol (\% lifetime) } \\
\hline Abuse & 15 & 30 & 47 & 38 & $2,3,4>I^{*}$ \\
\hline Dependence & 10 & 6 & 30 & 28 & $3,4>1,2^{* *}$ \\
\hline \multicolumn{6}{|l|}{ Poly-drug (\% lifetime) } \\
\hline Abuse & 0 & 0 & 0 & 0 & \\
\hline Dependence & 0 & 0 & 0 & 0 & \\
\hline \multicolumn{6}{|l|}{ METH+ groups } \\
\hline Age at first METH use (mean yrs) M (sd) & - & - & $23(9)$ & $25(7)$ & \\
\hline Total METH use (mean yrs) M (sd) & - & - & $10(6)$ & $7(6)$ & $3>4^{* *}$ \\
\hline Last use of METH (mean days) M (sd) & - & - & $125(106)$ & $176(330)$ & \\
\hline \multicolumn{6}{|l|}{ HIV+ groups } \\
\hline HIV RNA, plasma (log copies/mL) M (sd) & - & $3.5(1.1)$ & - & $3.5(1.2)$ & \\
\hline
\end{tabular}

Notes: $* p<0.05 ; * * p<0.005$.

Abbreviations: M, mean; sd, standard deviation; HIV, human immunodeficiency virus; METH, methamphetamine; WRAT4, Wide Range Achievement Test 4. 
Table 3 Distribution of genotypes and alleles by group*

\begin{tabular}{|c|c|c|c|c|}
\hline & Control & HIV only & METH only & METH and HIV \\
\hline Genotype n (\%) & $(n=49)$ & $(n=72)$ & $(n=50)$ & $(n=58)$ \\
\hline $\mathrm{Val} / \mathrm{Val}$ & $15(3 \mid)$ & $25(35)$ & $7(14)$ & $18(31)$ \\
\hline $\mathrm{Val} /$ Met & $23(47)$ & $38(53)$ & $34(68)$ & $28(48)$ \\
\hline Met/Met & II (22) & $9(I 2)$ & $9(18)$ & $12(2 \mid)$ \\
\hline Val & $53(54)$ & $88(6 I)$ & $48(48)$ & $64(55)$ \\
\hline Met & $45(46)$ & $56(39)$ & $52(52)$ & $52(45)$ \\
\hline HWE, $X^{2}$ ( $P$ value) & $0.09(0.95)$ & $0.49(0.77)$ & $3.79(0.15)$ & $0.01(0.99)$ \\
\hline
\end{tabular}

Notes: *There were no significant genotype or allele differences. Abbreviation: HWE, Hardy-Weinberg equilibrium.

on the executive functioning domain and background variables were analyzed using chi-squared tests.

\section{Results}

\section{Participant characteristics}

Participant characteristics by group membership are shown in Table 2. All four groups were comparable with regard to age and education. However, African-Americans were significantly less represented in the METH-only group compared to controls $(P=0.001)$, HIV-only $(P=0.02)$, and METH+/HIV+ $(P=0.001)$ groups. Lifetime diagnosis of major depression was significantly greater among those in the HIV-only $(P=0.006)$ and METH $+\mathrm{HIV}+(P=0.002)$ groups compared to controls. As expected, METH+ groups had higher rates of lifetime alcohol abuse $(P=0.004)$ and dependence $(P=0.001)$, cannabis abuse $(P=0.001)$ and dependence $(P=0.002)$, cocaine abuse $(P=0.001)$ and dependence $(P=0.0001)$, as well as hallucinogen abuse $(P=0.02)$ compared to METH- groups.
METH+ groups did not differ on cumulative days of METH use or length of abstinence; however, the METH-only group did report significantly more total years of METH use compared to the METH+/HIV+ group $(P=0.003)$. HIV+ groups did not differ on mean plasma HIV RNA levels $(P=0.795)$.

Genotype and allele distributions in the four groups revealed no significant differences and all groups were in Hardy-Weinberg equilibrium (Table 3). Furthermore, when examining differences between genotype and demographic variables the four groups were also comparable (Table 4). However, significant differences were observed in the control group for education $(P=0.02)$ and lifetime cannabis abuse $(P=0.05)$, in which carriers of the Met/Met genotype had one more year of education on average and no history of cannabis abuse, compared to Val allele carriers $(0 \%$ versus $27 \%$ ). In addition, among the METH-only group, $78 \%$ of Met/Met homozygotes versus $40 \%$ of Val allele carriers had a history of alcohol abuse $(P=0.04)$.

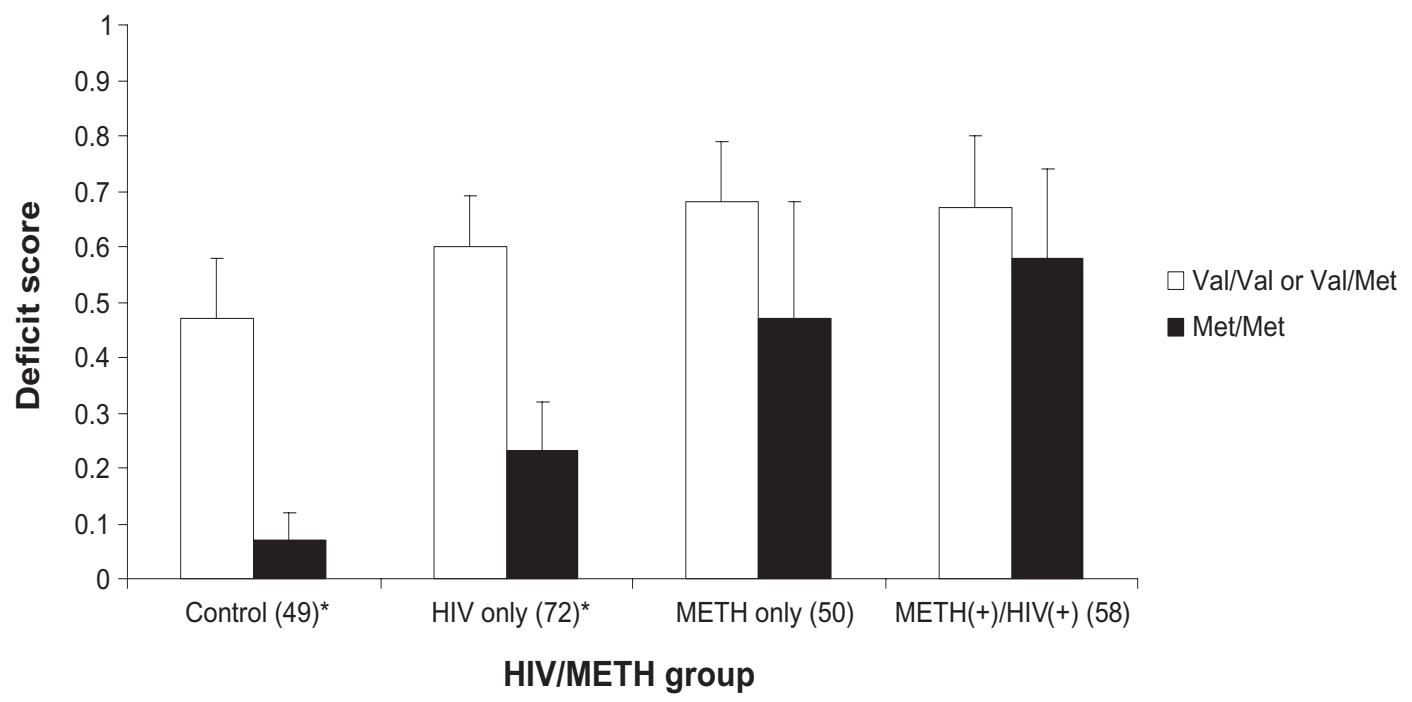

Figure I Executive functioning deficit Scores by HIV/METH Group and COMT Genotype. Notes: Bars represent mean deficit scores. *Statistically significant at $P<0.0$ I. 


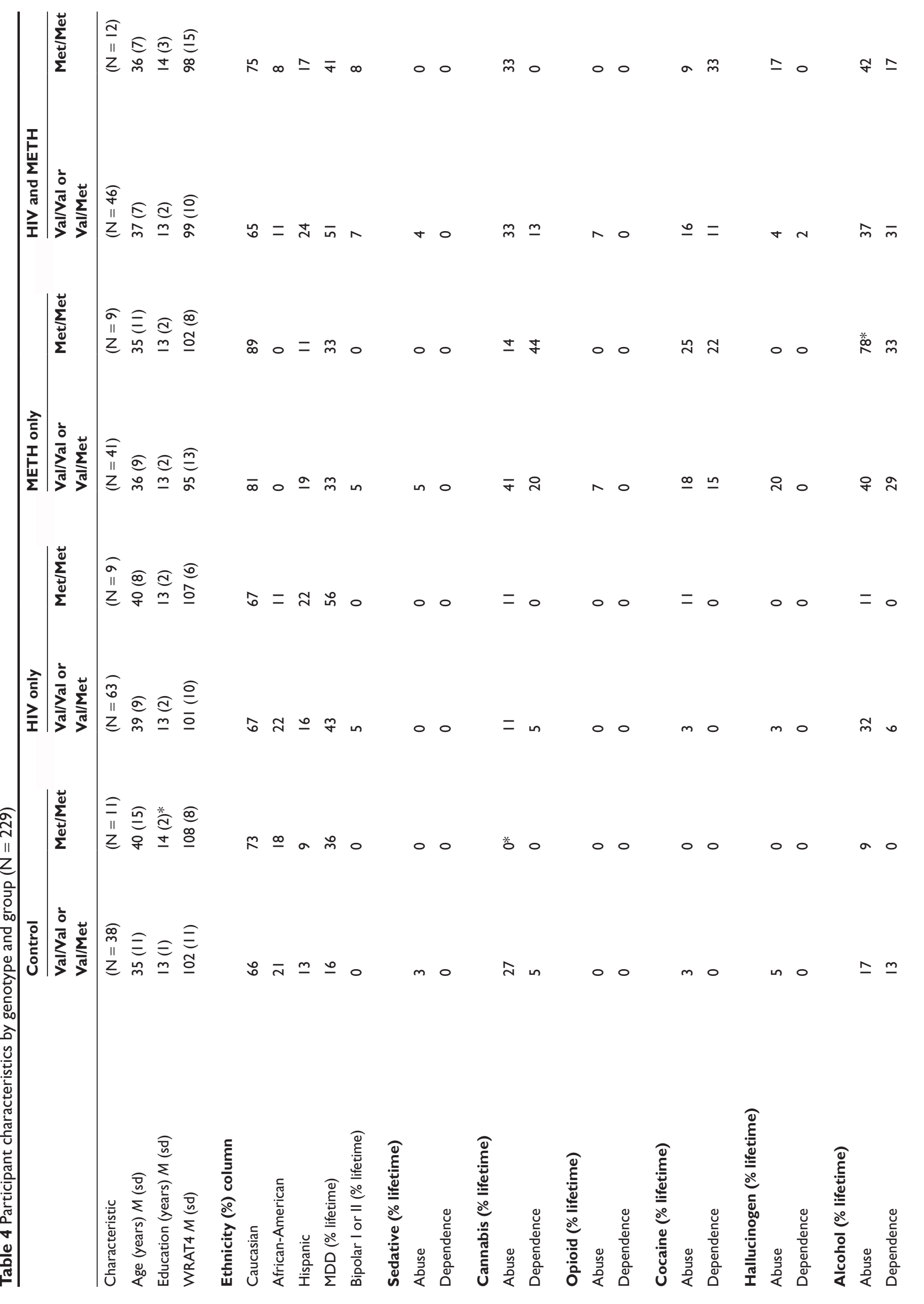




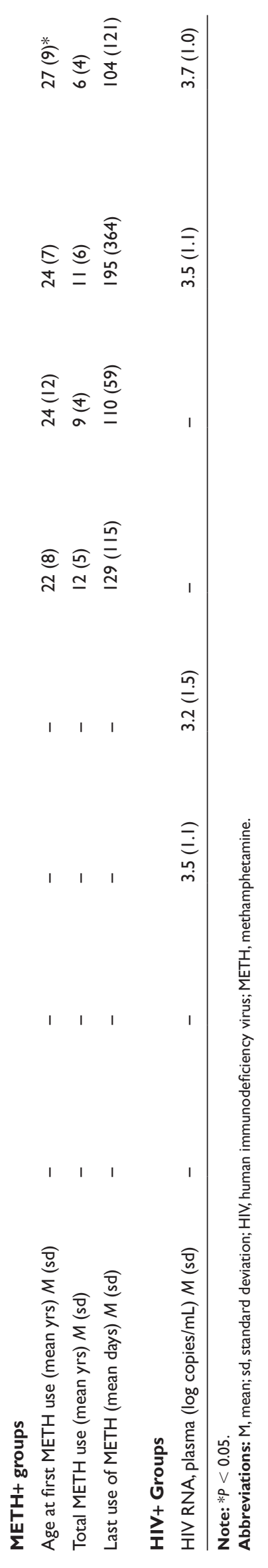

\section{COMT and executive functioning}

Executive functioning deficit scores (Figure 1) were significantly lower among Met/Met homozygotes in both the $\operatorname{control}(P=0.001)$ and HIV-only $(P=0.007)$ groups compared to Val allele carriers. However, the Met/Met genotype did not confer better executive functioning among METH-only $(P=0.458)$ or METH+/HIV+ $(P=0.733)$ groups. Similar results were found when examining rates of impairment among the four groups by genotype. The rate of impairment among Met/Met homozygotes was lowest for control participants (9\%) and greatest for METH+/HIV+ participants (58\%), while rates of impairment were generally similar among Val allele carriers across all groups (range $42 \%$ to $54 \%$ ). Analysis stratified by each of the three ethnic groups in our sample also produced similar results at the trend level, albeit not statistically significant (data not shown). As expected, Caucasians, our largest group, represented the monotonic trend illustrated in the full sample; whereas, the trend seen in the Hispanic and African-American groups, although similar to the full sample, was less pronounced, which may be a result of reduced power with these smaller subgroups.

\section{COMT and other neurocognitive domains}

COMT's association with speed of information processing (SIP), motor speed, learning, recall, verbal fluency, and working memory domains by each group are illustrated in Figure 2. Other than a statistically significant deficit score difference for verbal fluency, which can be considered to have an executive component, among those in the METHonly group $(P=0.002)$, no other significant results were found within any of these other neurocognitive domains. In addition, deficit score trends for Met/Met carriers across the four groups were not in accord with those found for executive functioning. The recall domain is a possible exception in that the monotonic trend observed for executive functioning among Met/Met homozygotes is present; however unlike executive functioning, the recall domain shows this same trend for Val allele carriers and thus provides little evidence of a genotype effect.

\section{Discussion}

To our knowledge, these preliminary findings are the first to demonstrate a context-dependent neuroprotective effect of the COMT Met/Met genotype on executive functioning in a sample of individuals with and/or without METH dependence and/or HIV-infection. Our results support previous work ${ }^{22-26}$ suggesting a neuroprotective effect, specifically in executive functioning, among relatively healthy individuals 


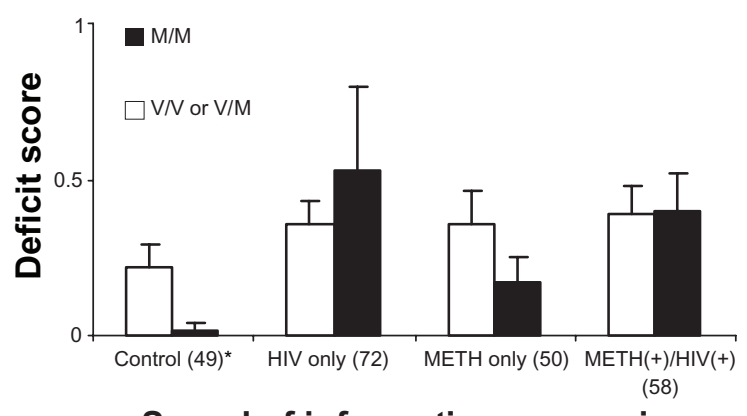

Speed of information processing
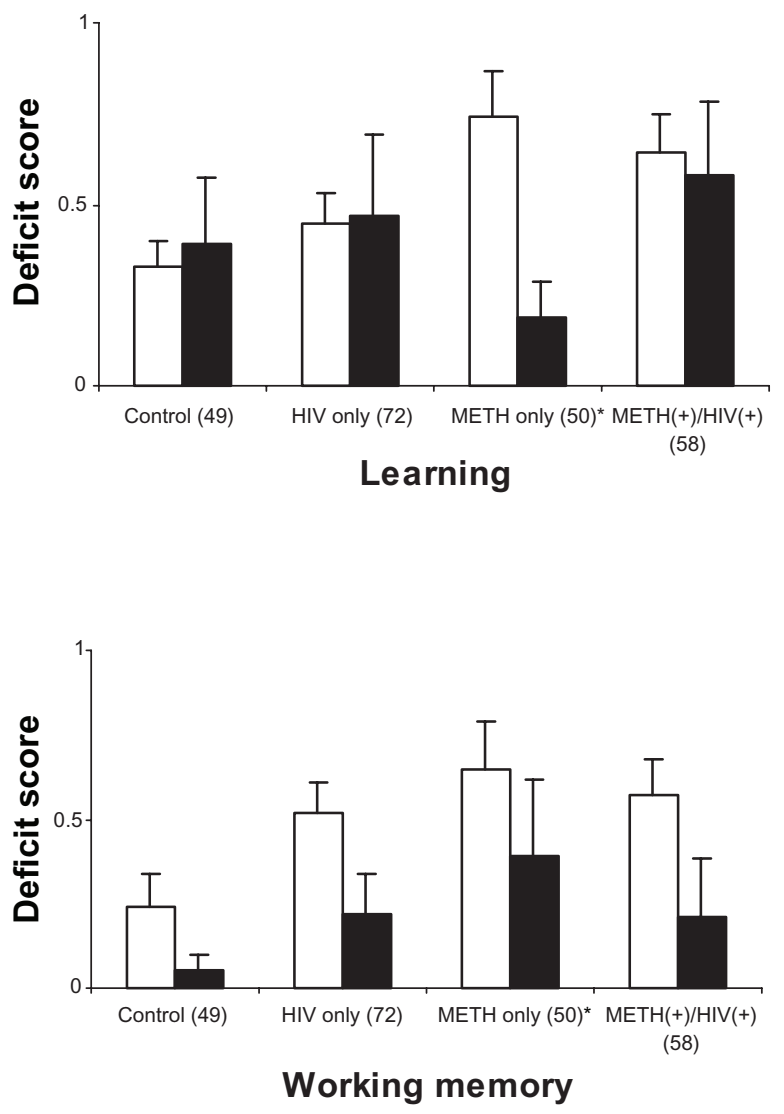
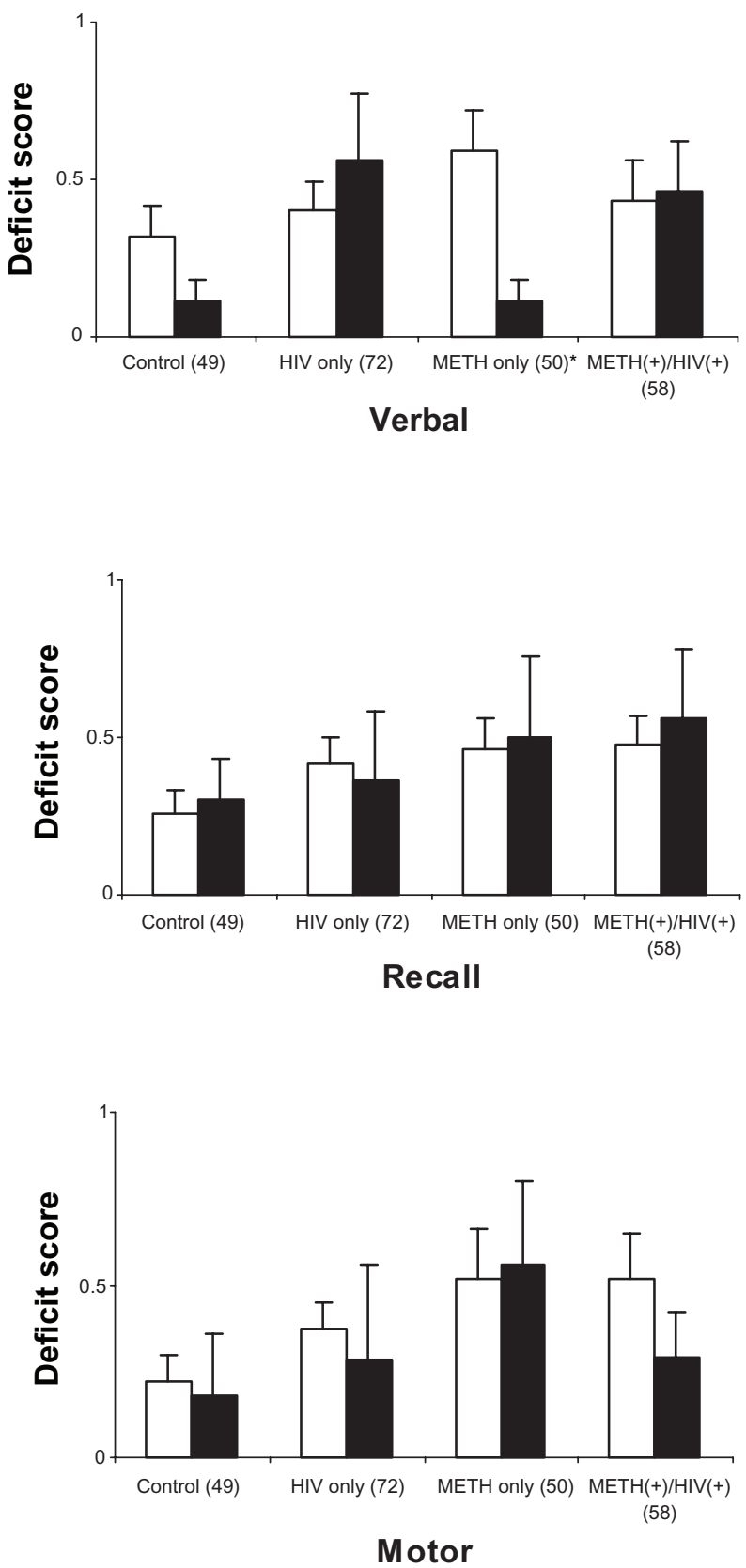

Figure 2 Deficit scores for speed of information processing, verbal fluency, learning, recall, working memory, and motor speed by HIV/METH group and COMT Genotype. Note: Dark bars represent Met/Met genotype and light bars represent Val/Val and Val/Met genotype.

*Statistically significant at $P<0.05$.

with the COMT Met/Met genotype. However, in this study we also demonstrate that this neuroprotective effect of the Met/Met genotype on executive functioning is attenuated among currently abstinent METH-dependent individuals, irrespective of their HIV-status. This finding is consistent with Mattay and colleagues ${ }^{29}$ who reported COMT interacts with acute amphetamine exposure in an inverted U-shaped fashion to produce harmful effects on cognitive performance among healthy individuals with a Met allele by disrupting dopamine levels as well as exceeding the critical threshold of dopamine signaling and associated processing load.

Our findings extend this previous work by demonstrating the putative context-dependent effect of the Met/Met genotype on executive functioning in a sample of abstinent, chronically exposed methamphetamine individuals with and without co-morbid HIV-infection. Thus, it appears that COMT not only interacts with acute amphetamine exposure resulting in executive functioning deficits but also may confer 
a lasting neurocognitive effect into early abstinence. Among $\mathrm{METH}+$ groups, it may be that the slower rate of dopamine clearance conferred by Met/Met genotype may increase the risk of adverse effects of METH consumption acutely and over time thereby making executive impairment comparable to that of Val allele carriers in the control group. It should be noted that noradrenaline is also metabolized by COMT and has been shown to effect executive functioning in animals (for detailed review see) ${ }^{33}$ and methamphetamine can be neurotoxic to noradrenaline pathways, albeit to date this has only been shown in the hippocampus. ${ }^{34}$ Thus, it is plausible that the effects we report here are in part mediated by noradrenergic mechanisms for which we did not control.

Our hypothesis that METH-dependent Val-carriers would show improved executive functioning compared to controls as a result of more efficient clearance of DA was not supported. In fact, across all groups within this study, executive functioning deficit scores and rates of impairment were comparable for Val-allele carriers. One potential explanation for this finding is that other polymorphisms on the COMT gene or elsewhere ${ }^{35}$ in the genome are confounding or compensating for the presumed unfavorable effect of the Val allele on executive functioning. In fact, previous work has shown that other loci within the COMT gene may affect phenotypic expression; ${ }^{36}$ thereby suggesting research into gene-gene (or locus-locus) interaction as well as haplotype effects on executive functioning among METH-dependent populations may be warranted. Thus, in partial accordance with Mattay and colleagues, ${ }^{29}$ the effect of COMT Met/Met activity on executive functioning may be dependent on where an individual lands on the inverted U-shaped curve given a particular environmental (eg, METH dependence) and/or genetic (eg, COMT genotype) context. As the current study did not formally measure dopamine signaling or account for other contextual factors, future research that includes measures of dopamine signaling (eg, fMRI) and examines other groups and contexts will better clarify the role of COMT on executive functioning. In particular, studies are needed which examine females and those of Asian ancestry, as well as other stimulants such as cocaine and other viral contexts such as Hepatitis $\mathrm{C}$ that were not examined in the current study.

For our secondary aim, we sought to estimate the extent of the specificity of the effect of the Val158Met polymorphism on executive functioning by examining other neurocognitive domains among the same sample. We found little evidence to support a similar relationship among speed of information processing, motor speed, learning, recall, verbal fluency, or working memory domains. Although Met/Met homozygotes, as well as carriers of a Val allele within the recall domain, appeared to show a similar monotonic trend to that shown in the executive functioning domain, it is likely that this parallel trend for both genotypes in the recall domain is a result of an environmental insult (eg, METH dependence, HIV-infection) rather than the Val158Met polymorphism. In fact, the deficit score trend for the recall domain among both genotypes across the four groups replicates previous findings of global neurocognitive impairment among METH-dependent and/or HIV-infected individuals. ${ }^{4}$ Surprisingly, we did not observe the full presumed relationship between COMT and working memory which is also largely dependent on the integrity of the PFC and has been demonstrated in previous work. ${ }^{29,35}$ Although this may represent a false-negative finding, another possible explanation for this discordance is the difference in tests used to estimate working memory. Mattay and colleagues ${ }^{29}$ and Raz and colleagues ${ }^{35}$ employed the N-back test whereas in the current study we used a battery of three tests (Table 1) that did not include the N-back test. Thus, it may be that the relationship between COMT and working memory is test-specific or less sensitive to deficits observable in early abstinence from METH. On the other hand, when we examined the relationship between COMT and our executive functioning domain consisting of three tests (including the Wisconsin Card Sort Test used by Mattay and colleagues) $)^{29}$ we observed concordance with Mattay and colleagues. ${ }^{29}$ In fact, examination of the three individual tests included in the executive functioning domain revealed all three tests demonstrated the trend in which the Met/Met genotype has a neuroprotective effect among control and HIV-only groups but is attenuated among METH+ groups (data not shown). Thus, our results provide preliminary evidence suggesting relatively strong specificity of the Val158Met polymorphism on tests of executive functioning.

Although these findings provide further insight into the relationship between $C O M T$ and neurocognitive functioning, specifically executive functioning, several limitations should be considered. The sample for our study was relatively small for a genotype-phenotype investigation. Although, in light of previous work ${ }^{27}$ examining COMT and neurocognition as well as the high minor allele (Met) frequency of the COMT Val158Met polymorphism with the populations under investigation $(>40 \%$ ), our total sample size for the HIV-only group provided adequate power $(1-\beta=0.82)$, albeit power for the other groups was slightly below optimal (Control: $1-\beta=0.67$; METH-only: $1-\beta=0.68$; HIV+/METH+: $1-\beta=0.74)$. To conserve power we did not adjust for several factors that may alternatively explain higher rates of executive dysfunction 
in our METH+ groups. Both METH+ groups had slightly lower WRAT-4 reading scores as well as higher rates of abuse/dependence on several substances. In addition, stratified analysis by the three ethnic groups resulted in a loss of power and subsequently inhibited statistical replication of the COMT-executive functioning relationship demonstrated in the full sample, albeit results were supportive in terms of the magnitude and direction of observed effects. Therefore, further validation of the relationship between the Val158Met loci and executive functioning is required among larger ethnically/ancestrally diverse groups ideally utilizing available ancestral informative markers (AIMs) for ancestral classification.

Despite the limitations, our findings suggest a specific context-dependent relationship between COMT and executive functioning. However, it is clear from previous work as well as the current study that the link between COMT and neurocognitive functioning is not fully understood and continued work in this area is needed. Future research in this area could potentially lead to relevant public health innovations such as personalized health promotion interventions that may assist in curbing the transmission of HIV, other sexually transmitted infections, and non-adherence to treatment regimens as well as pharmacological treatments for executive dysfunction in both healthy and vulnerable populations.

\section{Acknowledgements}

The authors wish to acknowledge support from the United States National Institutes of Health (grant numbers P01-DA12065 and P30-MH62512) and the contributions of study participants and staff at the HIV Neurobehavioral Research Center (HNRC), San Diego, CA, USA.

The HNRC Group is affiliated with the University of California, San Diego, the Naval Hospital, San Diego, and the Veterans Affairs San Diego Healthcare System, and includes: Director: Igor Grant, M.D.; Co-Directors: J Hampton Atkinson, M.D., Ronald J Ellis, M.D., Ph.D., and J Allen McCutchan, M.D.; Center Manager: Thomas D Marcotte, Ph.D.; Business Manager: Melanie Sherman; Naval Hospital San Diego: Braden R Hale, M.D., M.P.H. (P.I.); Neuromedical Component: Ronald J Ellis, M.D., Ph.D. (P.I.), J Allen McCutchan, M.D., Scott Letendre, M.D., Edmund Capparelli, Pharm.D., Rachel Schrier, Ph.D.; Jennifer Marquie-Beck; Terry Alexander, R.N.; Neurobehavioral Component: Robert K Heaton, Ph.D. (P.I.), Mariana Cherner, Ph.D., Steven Paul Woods, Psy.D., David J Moore, Ph.D.; Matthew Dawson, Donald Franklin; Neuroimaging Component: Terry Jernigan, Ph.D. (P.I.),
Christine Fennema-Notestine, Ph.D., Sarah L Archibald, M.A., John Hesselink, M.D., Jacopo Annese, Ph.D., Michael J Taylor, Ph.D., Neurobiology Component: Eliezer Masliah, M.D. (P.I.), Ian Everall, FRCPsych., FRCPath., Ph.D., Cristian Achim, M.D., Ph.D.; Neurovirology Component: Douglas Richman, M.D., (P.I.), David M Smith, M.D.; International Component: J Allen McCutchan, M.D., (P.I.); Developmental Component: Ian Everall, FRCPsych., FRCPath., Ph.D. (P.I.), Stuart Lipton, M.D., Ph.D.; Clinical Trials Component: J Allen McCutchan, M.D., J Hampton Atkinson, M.D., Ronald J Ellis, M.D., Ph.D., Scott Letendre, M.D.; Participant Accrual and Retention Unit: J Hampton Atkinson, M.D. (P.I.), Rodney von Jaeger, M.P.H.; Data Management Unit: Anthony C Gamst, Ph.D. (P.I.), Clint Cushman (Data Systems Manager), Daniel R Masys, M.D. (Senior Consultant); Statistics Unit: Ian Abramson, Ph.D. (P.I.), Florin Vaida, Ph.D., Christopher Ake, Ph.D.

\section{Disclosures}

The authors have no conflicts of interest that are directly relevant to this study. The views expressed in this article are those of the authors and do not reflect the official policy or position of the Department of the Navy, Department of Defense, nor the United States Government.

\section{References}

1. Anderson SW, Tranel D. Neuropsychological consequences of dysfunction in human dorsolateral prefrontal cortex. In: Handbook of Neurophysiology. Grafman J, editor. Vol 7. 2nd ed. Amsterdam: Elsevier; 2002. pp. 145.

2. Reger M, Welsh R, Razani J, Martin DJ, Boone KB. A meta-analysis of the neuropsychological sequelae of HIV infection. J Int Neuropsychol Soc. 2002;8(3):410-424.

3. Gonzalez R, Rippeth JD, Carey CL, et al. Neurocognitive performance of methamphetamine users discordant for history of marijuana exposure. Drug Alcohol Depend. 2004;76(2):181-190.

4. Rippeth JD, Heaton RK, Carey CL, et al. Methamphetamine dependence increases risk of neuropsychological impairment in HIV infected persons. J Int Neuropsychol Soc. 2004;10(1):1-14.

5. Scott JC, Woods SP, Matt GE, et al. Neurocognitive effects of methamphetamine: A critical review and meta-analysis. Neuropsychol Rev. 2007;17(3):275-297.

6. Grant I, Heaton RK, Atkinson JH. Neurocognitive disorders in HIV-1 infection. HNRC group. HIV neurobehavioral research center. Curr Top Microbiol Immunol. 1995;202:11-32.

7. Heaton RK, Grant I, Butters N, et al. The HNRC 500 - neuropsychology of HIV infection at different disease stages. HIV neurobehavioral research center. J Int Neuropsychol Soc. 1995;1(3):231-251.

8. White DA, Heaton RK, Monsch AU. Neuropsychological studies of asymptomatic human immunodeficiency virus-type-1 infected individuals. the HNRC group. HIV neurobehavioral research center. J Int Neuropsychol Soc. 1995;1(3):304-315.

9. Starr JM, Fox H, Harris SE, Deary IJ, Whalley LJ. COMT genotype and cognitive ability: A longitudinal aging study. Neurosci Lett. 2007;421(1):57-61.

10. Tunbridge EM, Harrison PJ, Weinberger DR. Catecholo-methyltransferase, cognition, and psychosis: Val158Met and beyond. Biol Psychiatry. 2006;60(2):141-151. 
11. McCann UD, Wong DF, Yokoi F, Villemagne V, Dannals RF, Ricaurte GA. Reduced striatal dopamine transporter density in abstinent methamphetamine and methcathinone users: Evidence from positron emission tomography studies with [11C]WIN-35,428. J Neurosci. 1998;18(20): 8417-8422.

12. Sekine $Y$, Iyo M, Ouchi Y, et al. Methamphetamine-related psychiatric symptoms and reduced brain dopamine transporters studied with PET. Am J Psychiatry. 2001;158(8):1206-1214.

13. Berger JR, Kumar M, Kumar A, Fernandez JB, Levin B. Cerebrospinal fluid dopamine in HIV-1 infection. AIDS. 1994;8(1):67-71.

14. Larsson M, Hagberg L, Forsman A, Norkrans G. Cerebrospinal fluid catecholamine metabolites in HIV-infected patients. J Neurosci Res. 1991;28(3):406-409.

15. Ellis R, Childers ME, Kumar AM, Kumar M, Goetz T, Caligiuri M. Decreased cerebrospinal fluid dopamine correlates with impaired motor skills in HIV-1 infection and methamphetamine dependence. San Diego, CA: Society for Neuroscience, 34th Annual Meeting; 2004 Oct 23-27.

16. Kumar AM, Fernandez J, Singer EJ, et al. Human immunodeficiency virus type 1 in the central nervous system leads to decreased dopamine in different regions of postmortem human brains. J Neurovirol. 2009; 15(3):257-274.

17. Carboni E, Silvagni A. Dopamine reuptake by norepinephrine neurons: Exception or rule? Crit Rev Neurobiol. 2004;16(1-2):121-128.

18. Westerink BH, Spaan SJ. Simultaneous determination of the formation rate of dopamine and its metabolite 3,4-dihydroxyphenylacetic acid (DOPAC) in various rat brain areas. Brain Res. 1982;252(2):239-245.

19. Li T, Chen CK, Hu X, et al. Association analysis of the DRD4 and COMT genes in methamphetamine abuse. Am J Med Genet B Neuropsychiatr Genet. 2004;129(1):120-124.

20. Chen J, Lipska BK, Halim N, et al. Functional analysis of genetic variation in catechol-O-methyltransferase (COMT): Effects on mRNA, protein, and enzyme activity in postmortem human brain. Am J Hum Genet. 2004;75(5):807-821.

21. Papaleo F, Crawley JN, Song J, et al. Genetic dissection of the role of catechol-O-methyltransferase in cognition and stress reactivity in mice. J Neurosci. 2008;28(35):8709-8723.

22. Egan MF, Goldberg TE, Kolachana BS, et al. Effect of COMT Val108/158 met genotype on frontal lobe function and risk for schizophrenia. Proc Natl Acad Sci U S A. 2001;98(12):6917-6922.

23. Joober R, Gauthier J, Lal S, et al. Catechol-O-methyltransferase val-108/158-met gene variants associated with performance on the wisconsin card sorting test. Arch Gen Psychiatry. 2002;59(7):662-663.
24. Malhotra AK, Kestler LJ, Mazzanti C, Bates JA, Goldberg T, Goldman D A functional polymorphism in the COMT gene and performance on a test of prefrontal cognition. Am J Psychiatry. 2002;159(4):652-654.

25. Rosa A, Peralta V, Cuesta MJ, et al. New evidence of association between $C O M T$ gene and prefrontal neurocognitive function in healthy individuals from sibling pairs discordant for psychosis. Am J Psychiatry. 2004;161(6): 1110-1112.

26. Bruder GE, Keilp JG, Xu H, et al. Catechol-O-methyltransferase $(C O M T)$ genotypes and working memory: Associations with differing cognitive operations. Biol Psychiatry. 2005;58(11):901-907.

27. Barnett JH, Jones PB, Robbins TW, Muller U. Effects of the catecholO-methyltransferase Val158Met polymorphism on executive function: A meta-analysis of the wisconsin card sort test in schizophrenia and healthy controls. Mol Psychiatry. 2007;12(5):502-509.

28. Barnett JH, Scoriels L, Munafo MR. Meta-analysis of the cognitive effects of the catechol-O-methyltransferase gene Val158/108Met polymorphism. Biol Psychiatry. 2008;64(2):137-144.

29. Mattay VS, Goldberg TE, Fera F, et al. Catechol O-methyltransferase val158-met genotype and individual variation in the brain response to amphetamine. Proc Natl Acad Sci U S A. 2003;100(10):6186-6191.

30. Carey CL, Woods SP, Gonzalez R, et al. Predictive validity of global deficit scores in detecting neuropsychological impairment in HIV infection. J Clin Exp Neuropsychol. 2004;26(3):307-319.

31. Glatt SJ, Faraone SV, Lasky-Su JA, Kanazawa T, Hwu HG, Tsuang MT. Family-based association testing strongly implicates DRD2 as a risk gene for schizophrenia in Han Chinese from Taiwan. Mol Psychiatry. 2009;14(9):885-893.

32. Zhang H, Morrison MA, Dewan A, et al. The NEI/NCBI dbGAP database: Genotypes and haplotypes that may specifically predispose to risk of neovascular age-related macular degeneration. BMC Med Genet. 2008;9:51.

33. Robbins TW, Roberts AC. Differential regulation of fronto-executive function by the monoamines and acetylcholine. Cereb Cortex. 2007; 17(Suppl 1):i151-i160.

34. Sweatt JD. Hippocampal function in cognition. Psychopharmacology (Berl). 2004;174(1):99-110.

35. Raz N, Dahle CL, Rodrigue KM, Kennedy KM, Land S. Effects of age, genes, and pulse pressure on executive functions in healthy adults. Neurobiol Aging. 2009 Jun 24 [Epub ahead of print].

36. Nackley AG, Shabalina SA, Tchivileva IE, et al. Human catechol-Omethyltransferase haplotypes modulate protein expression by altering mRNA secondary structure. Science. 2006;314(5807):1930-1933.
Neurobehavioral HIV Medicine

\section{Publish your work in this journal}

Neurobehavioral HIV Medicine is an international, peerreviewed, open access journal focusing on advances in research in HIV/AIDS, with specific reference to the neurological, psychiatric and behavioral consequences of the disease, concomitan infections and specific antiretroviral therapy. The manuscript

\section{Dovepress}

management system is completely online and includes a very quick and fair peer-review system, which is all easy to use. Visit http://www.dovepress.com/testimonials.php to read real quotes from published authors.

Submit your manuscript here: http://www.dovepress.com/journal-of-neurobehavioral-hiv-medicine-journal 